\title{
Long Noncoding RNAs as New Architects in Cancer Epigenetics, Prognostic Biomarkers, and Potential Therapeutic Targets
}

\author{
Didier Meseure, ${ }^{1}$ Kinan Drak Alsibai, ${ }^{1}$ Andre Nicolas, ${ }^{1}$ \\ Ivan Bieche, ${ }^{2}$ and Antonin Morillon ${ }^{3}$ \\ ${ }^{1}$ Platform of Investigative Pathology, Institute Curie, 75248 Paris, France \\ ${ }^{2}$ Department of Pharmacogenomics, Institute Curie and UMR 745 INSERM, University Descartes, 75248 Paris, France \\ ${ }^{3} U M R$ 3244, Non-Coding RNA, Epigenetics and Genome Fluidity, Institute Curie, 75248 Paris, France
}

Correspondence should be addressed to Didier Meseure; didier.meseure@curie.fr

Received 25 October 2014; Accepted 6 May 2015

Academic Editor: Konstantinos Arnaoutakis

Copyright (C) 2015 Didier Meseure et al. This is an open access article distributed under the Creative Commons Attribution License, which permits unrestricted use, distribution, and reproduction in any medium, provided the original work is properly cited.

\begin{abstract}
Recent advances in genome-wide analysis have revealed that $66 \%$ of the genome is actively transcribed into noncoding RNAs (ncRNAs) while less than $2 \%$ of the sequences encode proteins. Among ncRNAs, high-resolution microarray and massively parallel sequencing technologies have identified long ncRNAs ( $>200$ nucleotides) that lack coding protein function. LncRNAs abundance, nuclear location, and diversity allow them to create in association with protein interactome, a complex regulatory network orchestrating cellular phenotypic plasticity via modulation of all levels of protein-coding gene expression. Whereas lncRNAs biological functions and mechanisms of action are still not fully understood, accumulating data suggest that lncRNAs deregulation is pivotal in cancer initiation and progression and metastatic spread through various mechanisms, including epigenetic effectors, alternative splicing, and microRNA-like molecules. Mounting data suggest that several lncRNAs expression profiles in malignant tumors are associated with prognosis and they can be detected in biological fluids. In this review, we will briefly discuss characteristics and functions of lncRNAs, their role in carcinogenesis, and their potential usefulness as diagnosis and prognosis biomarkers and novel therapeutic targets.
\end{abstract}

\section{Long Noncoding RNAs and Functional Organization of the Genome}

1.1. Genome and Noncoding RNA. Large-scale genomic technologies (high-resolution microarray, whole genome, and RNA sequencing) combined with bioinformatics analyses have profoundly changed the genome organization understanding. Unexpectedly, these global transcriptional analyses revealed that $66 \%$ of the genome is transcribed and $80 \%$ presents biochemical marks of active transcription whereas less than $2 \%$ encodes proteins [1]. Discovery of a "second genetic code" consisting of ncRNAs has changed traditional concept of genomic organization characterized by presence of genes encoding islets scattered in a sea of repeats and nontranscribed proteins. Recently, high-throughput techniques have identified 58648 human lncRNAs [2].
1.2. Classification of Noncoding RNAs. NcRNAs are classified into two categories according to their structural or regulatory properties and size. This length, arbitrarily set to 200 nucleotides (nt), corresponds to the threshold of sensitivity of RNA extraction methods and can differentiate lncRNAs from short and medium ncRNAs such as microRNAs (miRNAs), small nucleolar RNAs (snoRNAs), and piwi RNAs (piRNAs) [3] (Table 1). Although functions of most ncRNAs are currently still largely uncharacterized, recent studies have involved them in mechanisms implicated in important biological functions and in various pathologic conditions, including neurodegenerative diseases and cancer $[4,5]$. It is likely that development of new diagnostic and prognostic classifications involving ncRNAs refine medical practice and that ncRNAs may be useful potential targets for novel anticancer therapies. In this review, we propose to 
TABLE 1: Type and main function of no-coding RNAs.

\begin{tabular}{|c|c|c|}
\hline Type & Size (nt) & Functions \\
\hline Short ncRNAs & $19-31$ & \\
\hline miRNAs & & $\begin{array}{l}\text { Targeting of mRNAs, regulation of proliferation, differentiation, and apoptosis } \\
\text { involved in human development }\end{array}$ \\
\hline siRNAs & & Posttranscriptional gene silencing; defense against pathogenic nucleic acids \\
\hline tiRNAs & & Regulation of transcription by targeting epigenetic silencing complexes \\
\hline piRNAs & & $\begin{array}{l}\text { Transposon repression, DNA methylation, development of germ cell, stem } \\
\text { self-renewal, and retrotransposon silencing }\end{array}$ \\
\hline tel-sRNAs & & Epigenetic regulation \\
\hline Mid-size ncRNAs & $\leq 200$ & \\
\hline snoRNAs & & rRNA modifications \\
\hline PASRs & & Regulation of the transcription of protein-coding genes \\
\hline TSSa-RNAs & & Maintenance of transcription \\
\hline PROMPTs & & Activation of transcription \\
\hline crasiRNAs & & Recruitment of heterochromatin and/or centromeric proteins \\
\hline Long ncRNAs & $>200$ & \\
\hline lincRNAs & & Involvement in biological processes such as dosage compensation and/or imprinting \\
\hline Intronic lncRNAs & & Possible link with posttranscriptional gene silencing \\
\hline T-UCRs & & $\begin{array}{l}\text { Regulation of miRNA and mRNA levels and antisense inhibitors for protein-coding } \\
\text { genes or other ncRNAs }\end{array}$ \\
\hline TERRAs & & Negative regulation of telomere length and activity through inhibition of telomerase \\
\hline Pseudogene RNAs & & Regulation of tumor suppressors and oncogenes by acting as microRNA decoys \\
\hline lncRNAs with dual functions & & Modulate gene expression through diverse mechanisms \\
\hline
\end{tabular}

summarize recent literature data on lncRNAs, their involvement in carcinogenesis, and their value as biomarkers and potential therapeutic targets.

1.3. Definition of $\operatorname{lncRNAs.~LncRNAs~transcripts~are~defined~}$ by a length greater than $200 \mathrm{nts}$ without any potential of translation [6]. This definition is however arbitrary and cannot now be based on a set of physical biochemical, structural or functional criteria. Length is not an absolute criterion because ncRNAs smaller than $200 \mathrm{nts}$ (BC1, snaR) are included in the lncRNAs subgroup. There is no specific transcription of lncRNAs, the transcriptional machinery being common to lncRNAs and mRNAs. Lack of reading frame cannot also be a discriminating factor between mRNAs and ncRNAs because $50 \%$ of lincRNAs contain regions with high translational potential, comparable to those of mRNAs, suggesting that they are actively exported to cytoplasm and translated into short polypeptides, albeit certainly not active. Noncoding characteristic is then not specific to lncRNAs, as bifunctional coding and noncoding lncRNAs and lncRNAs containing other ncRNAs (miRNAs, snoRNAs) have recently been discovered. Several mRNAs lose their ability to encode proteins (Xist) while others acquire coding function (SRA) [7]. Furthermore, by incorporating multiple exons from coding and noncoding genes, alternative splicing generates ambiguous transcripts beyond all classifications. Nevertheless, this definition appears currently to be the best that could be used waiting for a functional characterization of lncRNAs.
1.4. Investigative Methods and Annotation of $\operatorname{lncRNAs.~The~}$ first lncRNAs were discovered by using functional genetic approaches based on their relation to specific cellular mechanisms: (i) Ubxn transcriptional interference-induced repression by trithorax-promoted lncRNA $b d x$, (ii) sex chromosome dosage compensation and Xist-induced $\mathrm{X}$ chromosome, and (iii) inactivation and genomic imprinting promoted by antisense lncRNAs Airn and Kcnq1. These seminal studies established the concept of functional lncRNAs acting as transcriptional regulators involved in important and diverse biological processes. Over the past two decades, systematic global sequencing of cDNA libraries yielded comprehensive knowledge of the transcribed but noncoding component of the genome. These transcriptomic analyses have identified numerous lncRNAs. RNA immunoprecipitation and siRNAinduced inactivation techniques led to functional validation of many lncRNAs.

Currently, lncRNAs identification and functional characterization are based on an experimental approach combining (i) discovery of new noncoding transcripts using RNA-seq and ChIP-seq, (ii) annotation of transcribed regions extent and quantity of products transcribed through bioinformatic analysis, (iii) lncRNAs quantification in various cellular and tissue types and conditions, (iv) coexpression or coregulation studies, and (v) gain and loss functional tests on cell lines and xenografts (siRNAs, ASO, TALEN, and CRISPR/CAS9). Advantage of RNA-seq technique resides in its sensibility, allowing most weakly expressed lncRNAs to be identified. 
However, due to their frequent very low expression and complex exon/intron structures, it can be difficult to identify different transcripts produced from alncRNA gene. It is then necessary to use other techniques through epigenetic analyses (markers of promoter regions or entire gene), targeted transcripts of interest sequencing after capture on a DNA chip (capture-RNAseq) and $5^{\prime}$ and $3^{\prime}$ extremities identification analyses [8-11].

Systematic large-scale projects have contributed a lot to the systematic characterization of mammalian lncRNAs. For instance, the Fantom (Functional Annotation of the Mammalian genome) is an international project initiated by Japan in 2000 designed to identify and annotate entire transcripts of mouse. Results published identified 15000 lncRNAs in 2002, 23000 in 2005, and 34000 in 2006 with a transcribed genome fraction of $63 \%$. The ENCODE project initiated in 2003 is designed to identify, map, and make public all functional elements of the human genome. This project led to a catalog of lncRNAs with 10000 genes producing 15000 lncRNAs. Very recently, 58648 lncRNAs were identified by using the TCGA database, of which $79 \%$ were previously unannotated [2].

1.5. Properties of $\ln c R N A s$. LncRNAs genes outnumber those of short ncRNAs and are probably more abundant than mRNA genes. LncRNAs are very heterogeneous in size with some extending over tens of kilobases $(\mathrm{kb})$. They are transcribed in all regions of eukaryotic genome, particularly during development, and are remarkably specific for a given cellular and tissue type.

LncRNAs genes share many biochemical characteristics with proteins coding genes: predominant action of RNA polymerase II, epigenetic profiles, cotranscriptional modifications $\left(5^{\prime} \mathrm{Cap}\right.$, pre-lncRNAs alternative splicing, and $3^{\prime}$ polyadenylation), and exons and introns size. However, when lncRNAs are compared to mRNAs, they appear less stable and shorter, with fewer exons, a less well defined reading frame, and many repeat sequences (LINE, SINE). A minority group of poorly characterized lncRNAs is represented by nonpolyadenylated lncRNAs synthesized by RNA polymerase III and lncRNAs synthesized via alternative splicing and snoRNAs.

Depending on their relative position to the nearest coding genes, several categories of lncRNAs can be identified as follows.

(i) Intragenic Regions. Intragenic IncRNAs may be subdivided depending on how they overlap protein-coding genes or their orientation [45].

(a) Long intronic ncRNAs (linRNAs) constitute the major component of ncRNAs transcriptome. Unlike lincRNAs, tens of thousands linRNAs have recently been identified but only few have been analyzed functionally [46].

(b) Sense lncRNAs are transcribed from the sense strand of protein-coding genes containing exons. They may have some overlap with coding genes or cover entire sequence from an intron. If it has been shown that most have no protein-coding potential, several sense
lncRNAs can function as both ncRNAs and proteincoding genes (SRA, ENOD40).

(c) Antisense lncRNAs (NAT) represent 32\% of lncRNAs in humans and are poorly defined. They are transcribed from the antisense strand of protein-coding genes coding. They may also have overlapping with exonic or intronic regions or cover entire sequence from an intron. NATs are observed in many species, including animals, plants, yeasts, prokaryotes, and viruses, but have no sequence or conserved structure that could be indicative of a particular function despite several highly conserved lincRNAs [47].

(ii) Intergenic Regions. Intergenic lncRNAs (lincRNAs) are located in unannotated genomic regions and represent the best studied class of lncRNAs. LincRNAs are functional lncRNAs characterized by stability, K4H36 active domain of transcription, and tissue-specific expression. They act in trans and are primarily involved in epigenetic regulation of protein-coding genes expression and maintenance of stem cells pluripotency [48].

(iii) Enhancer ncRNAs (eRNAs). Two types of lncRNA (multiexonic or lacking introns with bidirectional transcription) are derived from enhancer sequences. Enhancer sequences regulate temporal and specific expression of genes via cis or trans mechanisms. The human genome contains approximately one million enhancer sequences most likely controlling the 19000 genes coding for proteins. LncRNAs derived from enhancer sequences play a crucial role in the formation of chromatin loops that allow stabilization and association of enhancer sequences with the promoter regions that initiate transcription of their target genes [49].

(iv) Promoters. PALR (promoter associated long ncRNA).

(v) Telomeric Regions. TERRA (telomeric repeat containing RNAs).

Within cells, lncRNAs are located in the nucleus, according to their main protein-coding genes epigenetic regulatory function. A minority of lncRNAs are involved in nucleocytoplasmic trafficking (NRON), mRNA stability, and translational regulation. Several lncRNAs (MALAT1) are cleaved by RNase $\mathrm{P}$ in a structural and regulatory intranuclear component (speckle) and a small cytoplasmic tRNA-like transcript. LncRNAs topography recognition should help in finding new efficient lncRNAs-based targeted therapies [50]. Finally, lncRNAs can be precursors of ncRNAs (snoRNA, miRNA).

1.6. Modular Organization of the Genome and Structural Plasticity of $\ln c R N A s$. The genome has a modular architecture composed of complex transcriptional loci characterized by close links between nucleotide sequences organized in sense/antisense and coding/noncoding transcripts. Thus, 
over $50 \%$ of protein-coding genes are associated with complementary antisense noncoding transcripts cis-regulating chromatin and adjacent genes expression although no systematic study classified them carefully as lncRNAs. Moreover, combined application of alternative splicing and transcriptional initiation and termination utilizes this modular architecture to ensure transcriptional diversification illustrated by the very large number of lncRNAs isoforms [48]. LncRNAs structural plasticity and biochemical properties better explain the great diversity of their mechanisms of action than their mere nucleotide sequence. Their organization in secondary and tertiary structures contributes to the creation of functional domains interacting with proteins (small ligands, multiprotein complexes) or hybridizing with nucleic acids (mRNA, miRNA, and DNA). Their interactions lead in turn to lncRNAs allosteric conformational changes allowing them to bind to other actors involved in gene expression and mRNA translation. Thus, lncRNAs initiate regulatory networks with high complexity at epigenetic, transcriptional, and posttranscriptional levels, in order to transmit and coordinate information flows in signaling pathways required for eukaryotic cells functioning [51].

1.7. Conservation, Evolution, and Origin of IncRNAs. LncRNAs have been observed in many eukaryotes. Conversely to protein-coding sequences, lncRNAs have rapidly evolved, explaining that lncRNAs orthologues to mammals are only found in vertebrates. Even in vertebrates, DNA sequences conservation is low and suggests that most lncRNAs are not functional due to insufficient selection pressure. Nonetheless, others criteria could be taken into account in assessment of their conservation, including genomic localization, transcriptional profile, and tertiary structure.

Current complexity of human physiology cannot be solely explained by expression of 20000 protein-coding genes, comparable to that observed in Drosophila melanogaster and Caenorhabditis elegans, but rather by parallel development of a noncoding genome. Unlike proteome, amount of ncRNAs has increased during evolution and it seems to be a significant correlation between lncRNAs expression levels and complexity, explaining that primates have most lncRNAs. The number of protein-coding genes cannot explain functioning of very finely regulated organs such as brain. This additional level of complexity observed in vertebrates may be partly related to expression of $\operatorname{lncRNAs}$ with a high spatiotemporal specificity and their interactions with DNA, mRNAs, and proteins. LncRNAs could then be considered as molecular sensors of environmental changes conferring evolutional plasticity that contributes to development of life complexity. During environmental changes, lncRNAs located within intergenic sequences may serve as supports for new functions allowing body to adapt new constraints. Protein-coding genes could generate transcripts from lncRNAs genes. Conversely, proteins may be synthesized from lncRNAs. Recent data have identified 24 human protein-coding genes with noncoding homologous genes in other species.

Recent emergence of numerous lncRNAs suggests that they continue to be actively synthesized (i) from ancestral genes that have lost their coding potential, (ii) by genes or other lncRNAs duplication, transposition, and mutation and (iii) de novo from intergenic DNA $[52,53]$.

1.8. LncRNAs Functions and Mechanisms of Action. Growing data have demonstrated that lncRNAs exhibit the greatest diversity among functional ncRNAs and play regulatory and structural roles in embryogenesis, stem cells pluripotency, allelic expression, protein-coding genes regulation, apoptosis, cycle control, growth, differentiation, and senescence. In practice, only a very limited number of lncRNAs (1\%) have been well characterized functionally in humans, including Xist, KCNQ10T1, AIR, HotAir, ANRIL, HOTTIP, MALAT1, TERRA, and HULC.

LncRNAs play a role in stem cells and differentiation: maintenance of pluripotency and lineage differentiation in embryonic stem cells (ESCs) and induced pluripotent stem cells (iPSCs). Adult stem cells are finely regulated by $\operatorname{lncRNAs}$ networks that are targets of most of master pluripotency transcription factors (Oct4, Sox2, Nanog, cMyc, and KLF4) [54].

LncRNAs are considered as crucial regulators coordinating protein-coding genes expression by numerous mechanisms depending on their cellular localization and leading to modifications of chromatin, transcription, and translation.

These regulatory mechanisms are located at epigenetic, transcriptional, posttranscriptional, and translational levels as follows.

(i) LncRNAs constitute a network of epigenetic modulators, recruiting, guiding, and forming platforms that build ribonucleoprotein complexes at specific genomic sites. $20 \%$ of human IncRNAs recruit multimolecular repressor, activator, or chromatin remodeling complexes (PRC1/PRC2 by ANRIL, LSD1/PRC2 by HOTAIR) whose subcatalytic units (EZH2, EED, BMI1, SUZ12, CBX7, CoREST, and JARID1) interact by altering histone code and methylation profile. Cis-acting lncRNAs adjacent to locus where they are transcribed act by transcriptional interference or by modifying chromatin. Transcriptional interference allows inhibition of preinitialization complexes and interaction with transcription factors. Chromatin modifications result from recruitment of complexes inhibiting (Polycomb by ANRIL) or activating (MLL by HOTTIP) gene expression. Trans-lncRNAs operate independently of complementarities sequences and act at distance on many genomic loci via specific DNA motifs. They influence genes expression by recruiting modifying chromatin complexes, binding transcriptional elongation factors, and inhibiting RNA polymerase [55].

(ii) LncRNAs associated with promoters (PaRNAs) and lncRNAs of enhancer type (eRNAs) can directly regulate transcription of target genes by transcriptional activation or suppression. PaRNAs function as protein coactivators facilitating gene transcription. PaRNAs of 50-200 nt are involved in repression of polycomb-targeted genes via mechanisms allowing transcription of these lncRNAs from common promoter regions to those of target genes regulated by these noncoding transcripts. Gene cis-repression is the result of PRC2 complex (SUZ12) recruitment by PaRNAs, attachment to promoter regions, and modification of histone methylation (H3K27me3) on target promoters. ERNAs have 
transcriptional activation functions of protein-coding genes coding, including genes involved in embryonic development and differentiation ( $n c R N A a 3$ and TAL, ncRNAa7 and SNAI1) [56-58].

(iii) LncRNAs can also act at posttranscriptional level and are widely involved in biogenesis, stability, and transcriptional activity of mRNAs. They regulate alternative splicing, promote trafficking, direct cellular localization, and promote mRNAs degradation. LncRNAs also synthesize miRNAs and build sponge-like structures to prevent binding of miRNAs to their target mRNAs (CDR1-as/ciRS-7, circular RNA sponge for $m i R-7)$ [59].

(iv) LncRNAs can finally bind to inhibiting factors of translation, interact with ribosomes, and allow transport of proteins.

LncRNAs may participate in assembly of specialized intranuclear functional structures, including speckles (MALAT1) paraspeckles (NEAT1), and polycomb body (TUG1).

Several lncRNAs, particularly lincRNAs, interact with factors (Oct4, Sox2, Nanog, c-Myc, Klf4, Smad, and Tcf3) and play key roles in maintaining stem cells pluripotency.

Moreover, lncRNAs have four known molecular functions (Figure 1): (1) Signal: lncRNAs regulate transcriptional activity or pathways (lincRNA-p21); (2) Guide: lncRNAs link specific proteins belonging to chromatin remodeling complexes and recruit them to homology containing target loci where genes silencing is promoted (HOTTIP, XIST); (3) Decoy: lncRNAs bind and titrate away proteins or RNAs. In the nucleus, they can bind transcription factors or DNA methyltransferase 1 (PANDA, Gas5, MALAT1), whereas in the cytoplasm, they can function like a sponge to attract proteins and miRNA/RISC complexes from their miRNA targets; (4) Scaffold: IncRNAs constitute adaptors that bind molecular complexes and regulate gene expression (HOTAIR, ANRIL, and TERC/TERT) $[18,60]$.

1.9. IncRNAs and Cancer. Because lncRNAs are involved in various and important physiological processes, their dysfunction should have important consequences for cell homeostasis. Several recent studies have indeed shown that expression of many lncRNAs varies significantly in different conditions compared to healthy tissue. Significance of these deregulations (consequence of a globally altered transcriptional status or causative and driving abnormality) is still a matter of debate. However, transcripts of noncoding genome revealed new dimension of the molecular architecture of cancer and lncRNAs are involved in all stages of oncogenesis. Gene expression profiles analysis of various tumors showed that lncRNAs are deregulated and functional studies have demonstrated that lncRNAs are implicated in general mechanisms of carcinogenesis. Moreover, genetic studies have revealed existence of mutations in their primary sequences. Since most genetic variants identified by genome-wide association studies (GWAS) are located outside coding genes, many of these mutations may therefore affect lncRNAs.

LncRNAs may regulate signaling pathways involved in initiation, tumor progression, and metastatic spread similar to protein-coding oncogenes and oncosuppressors. PINC and PGEM1 were the first oncogenic lncRNAs found overexpressed in breast and prostate carcinomas. Since then, many other lncRNAs have been identified, with oncogenic properties (KRASP, HULC, HOTAIR, MALAT1, HOTTIP, $A N R I L$, and RICTOR) or oncosuppressive properties (MEG3, GAS5, LincRNA-p21, PTENP1, TERRA, CCND1/CyclinD1, and TUG1). Interestingly, several lncRNAs may show both oncogenic and oncosuppressive activities, depending on cellular context. XIST noncoding transcript is overexpressed in male tumors and underexpressed in female tumors.

\section{(i) Oncogenic lncRNAs}

(a) SRA (steroid receptor RNA activator) is a coactivator for steroid receptors and acts as an ncRNA found in the nucleus and cytoplasm. SRA regulates gene expression mediated by steroid receptors through complexing with proteins also containing steroid receptor coactivator 1 (SRC-1). The SRA1 gene can also encode a protein that acts as a coactivator and corepressor. SRA levels have been found to be upregulated in breast tumors where it is assumed that increased SRA levels change the steroid receptors' actions, contributing to breast carcinogenesis. While expression of SRA in normal tissues is low, it is highly upregulated in breast, uterus, and ovary carcinomas [61-63].

(b) HOTAIR (HOX antisense intergenic RNA) is a long intergenic noncoding RNA with a length of $2.2 \mathrm{~kb}$ in HOXC locus and transcribed in antisense manner. HOTAIR regulates gene expression by modulating chromatin structures. It was the first lncRNA discovered to be involved in carcinogenesis. Polycomb group proteins mediate repression of transcription of thousands of genes that control differentiation pathways during development, pluripotency, and cancer progression. Target of PRC2 is HOXD locus on chromosome 2 where PRC2 in association with HOTAIR promotes transcriptional silencing of metastasis suppressor genes. HOTAIR acts as a molecular scaffold remodeling chromatin by causing histone modifications on target genes. HOTAIR comprises two known chromatin modification complexes with $5^{\prime}$ region binding to PRC2 complex responsible for repressive $\mathrm{H} 3 \mathrm{~K} 27$ methylation and $3^{\prime}$ region binding to LSD1, which initiates activating $\mathrm{H} 3 \mathrm{~K} 4$ demethylation $[64,65]$.

(c) ANRIL (antisense ncRNA in the INK4 locus) is a natural antisense transcript, which activates the two polycomb repressor complexes PRC1 and PRC2, resulting in chromatin reorganization with silencing of INK $4 b-A R F-I N K 4 a$. This results in p15/INK $4 \mathrm{~b}$, p14/ARF, and p16INK4a inhibition which are normally implicated in cell cycle negative regulation, senescence, and stress-induced apoptosis. ANRIL overexpression in prostate carcinomas has shown silencing of INK4b-ARF-INK4a and p15/CDKN2B. 


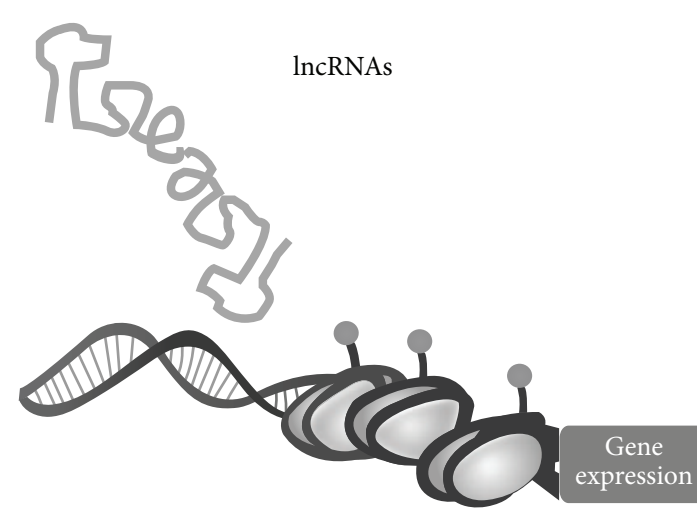

(a) Signal: lncRNAs affect expression of the downstream gene and regulate transcriptional activity or pathway

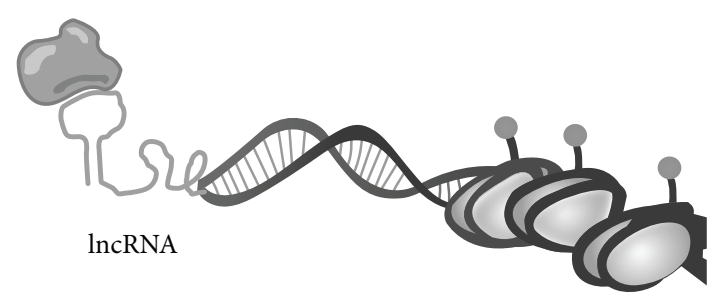

(c) Guide: IncRNAs can recruit chromatin modifying enzymes to target genes and function as guides

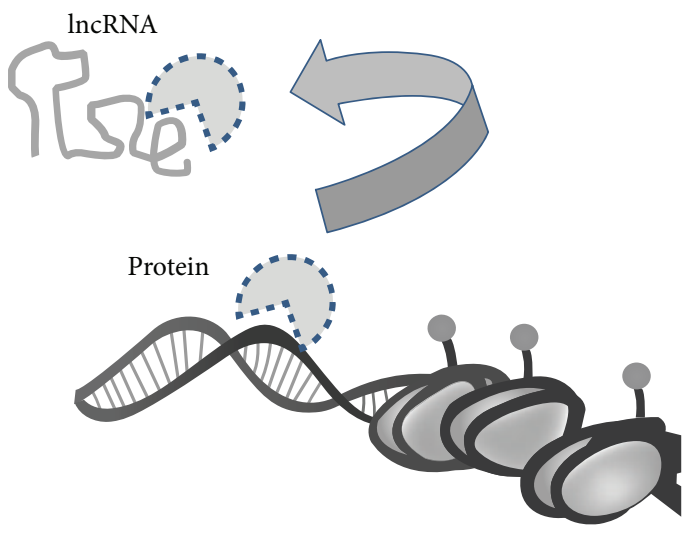

(b) Decoy: IncRNAs titrate proteins away from chromatin, or they can function as decoy for miRNA target sites

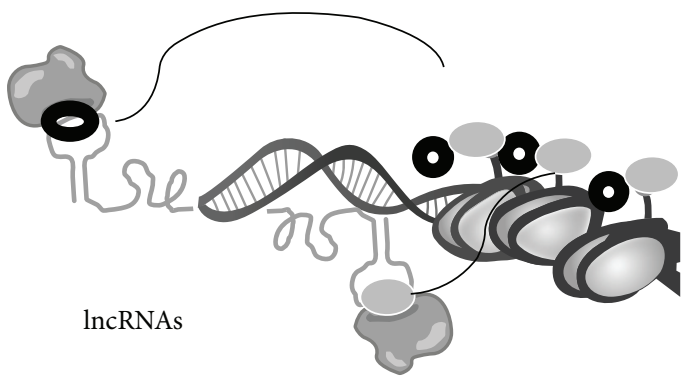

(d) Scaffold: IncRNAs can bring together multiple proteins to form ribonucleoprotein complexes

FIGURE 1: Molecular functions of lncRNAs.

Repression mechanism is mediated by direct binding to CBX 7 and SUZ12, 2 members of PRC1 and PRC2, respectively [66-68].

(d) MALAT1 (metastasis-associated lung adenocarcinoma transcript 1) is a lincRNA widely expressed in normal human tissues and overexpressed in a variety of breast, colon, liver, pancreas, prostate, and uterus carcinomas. MALAT1 locus at 11q13.1 has been identified to harbor chromosomal translocation break points and mutations in breast and bladder carcinomas. It is localized in nuclear speckles and recent studies have implicated MALAT1 in splicing, EMT, cell mobility, ECM remodeling, and metastatic spread [69].

(e) HULC (highly upregulated in liver cancer) has been suggested to act as a "sponge" that inhibits miR-372 by sequestering it away from potential mRNA targets [70].

(f) PCGEM1 (prostate cancer gene expression marker 1) is overexpressed in prostate cancer cells that promotes tumor cells initiation and progression and protects against chemotherapy-induced apoptosis. Moreover, the reciprocal negative control relationship between PCGEM1 and miR-145 regulates both prostate cancer cells proliferation and tumor growth. These results identify PCGEM1 and associated regulators as possible targets for prostate cancer therapy [71]. (ii) Tumor Suppressor lncRNAs

(a) MEG3 (maternally expressed gene 3) is a transcript of the maternally imprinted gene normally expressed in pituitary cells. MEG3 loss of expression is observed in pituitary adenomas and meningiomas. MEG3 acts by regulating p53 pathway. P53 levels are usually extremely low due to its rapid degradation via ubiquitin-proteasome pathway. P53 ubiquitination is mainly mediated by E3 ubiquitin ligase MDM2. MEG3 downregulates MDM2 expression, increases $\mathrm{p} 53$ protein level, stimulates p53-dependent transcription, and enhances p53 binding to target promoters [72].

(b) GAS5 (growth arrest-specific 5) is widely expressed in embryonic and adult tissues. GAS5 acts as a starvation or growth arrest-linked riborepressor for glucocorticoid receptors by inhibiting association of these receptors with their DNA recognition sequence. This suppresses several responsive genes activation including gene encoding cellular inhibitor of apoptosis 2 (cIAP2). GAS5 low expression levels have been observed in prostate and breast carcinomas $[73,74]$.

(c) CCND1/Cyclin D1 is transcribed from the promoter region of the Cyclin D1 gene. Cyclin D1 is a cell cycle regulator frequently mutated, amplified, and overexpressed in carcinomas. CCND1/Cyclin D1 recruits the RNA-binding protein TLS, which undergoes 
allosteric modification, resulting in Cyclin D1 gene inhibition $[75,76]$.

(d) LincRNA-p21 expression is directly induced by p53 signaling pathway. This lncRNA is implicated in global repression of genes interfering with p53 function to regulate cellular apoptosis [77].

(e) TERRA (telomeric repeat containing RNA) is expressed at chromosome ends. TERRA upregulation upon experimental manipulation or in ICF (immunodeficiency, centromeric instability, and facial anomalies) patients correlates with short telomeres. TERRA transcription facilitates the $5^{\prime}-3^{\prime}$ nuclease activity of Exol at chromosome ends, providing a means to regulate the telomere shortening rate. Thereby, telomere transcription can regulate cellular lifespan through modulation of chromosome end processing activities [78].

\section{(iii) Oncogenic and Tumor Suppressor lncRNAs}

(a) H19 is expressed from the maternal allele and has a pivotal role in genomic imprinting during cell growth and development. The locus contains H19 and IGF2, which are imprinted. This leads to differential expression of both genes $\mathrm{H} 19$ from maternal and IGF2 from paternal allele. This lncRNA presents both oncogenic and suppressive properties although the exact mechanism is still elusive [79].

LncRNAs are implicated in cancer epigenetics. Epigenetics refers to events that modulate activity of the genome without changing its sequence. It provides control of genome expression and establishment of tissular and cellular-type specific genes expression profiles. The main epigenetic mechanisms comprise chemical modifications of DNA and histones (cytosine methylation, posttranslational modifications of histones, chromatin remodeling, and nucleosome positioning). These chemical changes are reversible and controlled by enzyme complexes directly connected to metabolic and signaling pathways as well as sensors of extra- and intracellular microenvironments. Deregulation of these epigenetic mechanisms has been demonstrated in cancer cells which have no transcriptional gene or protein expression while being free of DNA damage. Carcinogenesis is very frequently associated with abnormal signaling and epigenetic abnormalities, most of which are considered as significant oncogenic events (aberrant methylation of oncosuppressor genes). LncRNAs interact with numerous proteins and in particular epigenetic regulators, including DNA methyltransferases and enzymatic complexes modifying chromatin and nucleosomes [80].

(i) Methylation of DNA was first observed in cancer epigenetic alterations. Cancer epigenome is characterized by global hypomethylation leading to genomic instability and hypermethylation of $\mathrm{CpG}$ islands which cause inactivation of genes oncosuppressors implicated in signaling pathways (DNA repair, apoptosis, and cycle cell regulation) or transcription factors involved in the control of these genes. LncRNAs bind to DNA methyltransferase, guide it to promoter of oncosuppressor genes, and ensure transcriptional silencing. (ii) Nucleosomes can be destabilized and restructured by remodeling complexes belonging to 4 families (SWI/SNF, ISWI, CHD, and INO80). Nucleosomes positioning and remodeling regulate gene expression by perturbating accessibility of DNA regulatory sequences by transcription factors and transcription machinery. Mutations and silencing of subunits of these complexes in various types of cancer have been observed. LncRNAs could reposition nucleosomes by interacting and guiding these remodeling complexes to specific genomic regions where nucleosome restructuring would ensure repression of oncosuppressive genes [80].

(iii) LncRNAs recruit histone modifying enzymes acting near the transcription site of lncRNAs. Histones modifying enzymes read, add, or remove covalent links with change in accessibility of chromatin and fixation of nonhistone protein effectors that decode modified histone code. Alterations in the expression of histone-modifying enzymes (mutations, overexpression) were observed in various carcinomas. These alterations inhibit oncosuppressive genes transcription (EZH2 histone methyltransferase responsible for repressive H3K27me3 mark and silencing of p15, p16, and p19; histone deacetylase responsible for loss of lysine acetylated H4) or activate oncogenes transcription (demethylases and deacetylases) [81].

Growing numbers of LncRNAs are implicated in numerous mechanisms characterizing hallmarks of cancer. They can act on proliferation via coactivation (SRA1) and inhibition of elongation (RN7SK). LncRNAs allow escape mechanisms from suppressor pathways via competition (PFS), silencing (ANRIL), or ribonucleic repression (GAS5). Several lncRNAs are involved in replicative immortality by inhibiting telomerase (TERRA). LncRNAs promote neoangiogenesis is by inhibiting HIF1A (AHIF, MALAT1). They induce resistance to cell death by acting on p53 and p21 (PCGEM1) and by reducing expression of proapoptotic genes (PANDA). Finally lncRNAs are involved in mechanisms of invasion and metastasis via deregulation of alternative splicing (MALAT1) or trans-silencing of HOXD locus (HOTAIR).

\section{LncRNAs as Biomarkers and Therapeutic Targets in Future Medical Practice}

LncRNAs are emerging as integral functional components of human genome and are now considered as critical regulators in molecular biology of cancer. Recent data have demonstrated that lncRNAs are associated with cancer initiation, tumor progression, and metastatic spread. Unlike mRNAs used as diagnostic and prognostic biomarkers while being expressed by numerous subtypes of malignant tumors, lncRNAs present cellular and tissular specificity and could serve as biomarkers and therapeutic targets in cancer. Furthermore, lncRNAs secreted or released by apoptotic or necrotic tumor cells can be detected in blood, plasma, and urine. There seems to be a significant correlation between levels of circulating nucleic acids and genomic, epigenetic, or transcriptional alterations associated with malignant tumors (Table 2).

(i) Esophagus. Three lncRNA signatures have been identified recently in oesophageal squamous cell carcinoma, 
TABLE 2: Representative lncRNAs involved in carcinogenesis and potential cancer biomarkers.

\begin{tabular}{llr}
\hline Cancer type & lncRNAs & References \\
\hline Esophagus & ENST00000435885.1, XLOC_013014, & ENST00000547963.1 \\
Stomach & GCAT1, H19, SUMO1P3 & {$[12]$} \\
Colon and rectum & HOTAIR, uc.73 & {$[13-17]$} \\
Liver & HULC, HOTAIR, MALAT1, HOTTIP, HEIH & {$[20-22]$} \\
Lung & MALAT1, TUG1, BANCR, GAS5 \\
Breast & HOTAIR, LincRNA-RoR, UCA1 \\
Ovary & HOTAIR \\
Bladder & UCA1, H19, Linc-UBC1, MALAT1 \\
Prostate & PCA3, PCAT1, PCGEM1 \\
Glioma & H19 \\
Melanoma & BANCR \\
Oral cavity and nasopharynx & HOTAIR, lnc-C22orf32-1, lnc-AL355149.1-1 \\
\hline
\end{tabular}

including ENST00000435885.1, XLOC_013014, and ENST00000547963.1. The expression of these lncRNAs classified the patients into two groups with significantly different overall survival [12].

(ii) Stomach. HOTAIR, GCAT1 (gastric cancer-associated transcript 1), H19, and SUMO1P3 (small ubiquitin-like modifier SUMO 1 pseudogene 3) are the main lncRNAS reported as overexpressed in gastric carcinomas. They are often associated with lymph node and distant metastasis, suggesting that they might serve as potential diagnostic and prognostic biomarker [13-17]. Moreover, CCAT1 expression is closely related to c-Myc activation [14]. CCAT1 functions as an oncogene and may be used as biomarker and potential therapeutic target in gastric carcinoma $[15,16]$.

(iii) Lower Digestive Tract. HOTAIR overexpression is observed in colorectal carcinomas with advanced stage and liver metastases [19]. uc.73 lncRNA is also associated with poor overall survival in patient with colorectal carcinomas [18]. These results place HOTAIR and uc.73 lncRNA as reliable biomarkers for poor prognosis in colorectal cancer.

(iv) Liver. In hepatocellular carcinomas (HCC), HULC (highly upregulated in liver cancer) was the first lncRNA with highly specific upregulation detected in blood [20]. High plasma HULC rates were observed in patients with high grades $\mathrm{HCC}$ or with $\mathrm{HBV}+$ status [21]. Recent data have shown that $\mathrm{HBx}$ could regulate HULC promoter to induce HCC via oncosuppressor p18 silencing [22]. $\mathrm{HBx}$ was found to downregulate lncRNA-Dreh, which can inhibit hepatocellular growth and metastasis in vitro and in vivo.

MALAT-1 and HOTAIR have been shown to be overexpressed in large cohorts of HCC patients. Furthermore, HOTAIR is a prognostic biomarker for recurrence after liver transplantation. SiRNA-mediated inhibition of
MALAT-1 and HOTAIR suppresses cancer cell viability and invasion, sensitizes TNF- $\alpha$, induces apoptosis, and increases chemotherapeutic sensitivity of HCC to cisplatin and doxorubicin [82, 83]. HOTTIP (HOXA transcript at the distal tip) and HOXA13 were also found to be upregulated in HCC. HOTTIP and HOXA13 levels were associated with HCC tumor progression, metastasis, and survival [84]. HEIH (high expression in HCC) is another lncRNA overexpressed in HCC and an independent prognostic factor associated with recurrence [85].

(v) Lung. MALAT1 is a key prognostic biomarker for metastatic spread in lung adenocarcinomas [23]. TUG1 (taurine upregulated gene 1) is generally downregulated in nonsmall cell lung carcinomas (NSCLC). In NSCLC patients, TUG1 low expression was associated with high TNM stage, tumor size, and poorer overall survival. Univariate and multivariate analyses revealed that TUG1 expression serves as an independent predictor for overall survival [24]. HOTAIR was initially reported to be highly expressed in NSCLCs with advanced stage and lymph node metastasis [25]. But more recent meta-analyses study showed that it did not reach statistical significance, and thus it needs further investigations [26]. In NSCLCs, BANCR (BRAF activated noncoding RNA) expression is significantly decreased compared to normal tissues. BANCR underexpression is considered as an independent prognostic factor and is associated with larger tumor size, advanced pathological stage, metastasis distance, and shorter overall survival. Recently, BANCR overexpression was found to play a key role in epithelial-mesenchymal transition [27]. In malignant pleural mesothelioma (MPM), GAS5 (growth arrest specific transcript 5) underexpression was observed compared to normal mesothelial tissue. Conversely, GAS5 was upregulated upon growth arrest induced by inhibition of Hedgehog and PI3K/mTOR signaling in MPM models [28]. 
(vi) Breast. Breast cancer progression is correlated with HOTAIR activity in numerous recent studies. The study carried out by Chisholm and colleagues demonstrated HOTAIR overexpression in primary and metastatic breast carcinoma tissues by using RNA in situ hybridization technique [29]. Another study focusing on HOTAIR revealed the dependence of HOTAIR expression on oestradiol production, due to its promoter region via several estrogen response elements. This study also demonstrated that HOTAIR knockdown induced apoptotic pathways in breast cancer cell lines and suggested estrogen receptors as coregulators for HOTAIR expression [30]. Conversely, a recent study focusing on 348 primary breast carcinomas revealed that increased DNA methylation led to HOTAIR downregulation and an unfavorable disease state, questioning suitability of HOTAIR as negative prognostic biomarker in breast carcinomas [31]. LincRNA-RoR suppresses p53 in response to DNA damage through interaction with heterogeneous nuclear ribonucleoprotein I (hnRNP I). Recent data demonstrated that hnRNP I can also form a functional ribonucleoprotein complex with UCA1 (urothelial carcinoma-associated 1) and increase UCA1 stability. Of interest, the phosphorylated form of hnRNP I, predominantly located in the cytoplasm, is responsible for the interaction with UCA1. Although hnRNP I enhances p27 translation through interaction with the $5^{\prime}$-untranslated region of p27 mRNAs, interaction of UCA1 with hnRNP I suppresses $\mathrm{p} 27$ protein expression via competitive inhibition. In support of this finding, UCA1 seems to have an oncogenic role in breast carcinogenesis both in vitro and in vivo. Finally, a negative correlation between p27 and UCA1 was found. Together, these results suggest an important role of UCA1 in breast carcinogenesis [32].

(vii) Glioma. H19/miR-675 signaling was recently identified as a critical pathway in glioma progression. By analyzing gene expression data, H19 increased levels of expression were found in high grade glioma. SiRNA-induced H19 depletion inhibited invasion in glioma cells. Furthermore, H19 expression was positively correlated with miR-675 and H19 inhibition reduced miR-675 expression. Collectively, these data suggest that $H 19$ regulates glioma development by deriving miR-675 [40].

(viii) ENT Tumors. Recently, HOTAIR was suggested to play a part in nasopharyngeal carcinoma (NPC). HOTAIR is implicated in NPC progression and patients with high HOTAIR levels have poor clinical outcome with tumor recurrence and distant metastasis $[42,43]$. In primary NPCs, upregulation of $\operatorname{lnc}$-C22orf32-1, Inc-AL355149.1-1, and lnc-ZNF6741 has been also observed. High levels of $\operatorname{lnc}$-C22orf32-1 and lnc-ZNF674-1 are associated with advanced tumor stages. Recurrent NPC displayed a distinctive lncRNA expression pattern with increased expression of $\operatorname{lnc}$-BCL2L11-3 and decreased expression of $\operatorname{lnc}$-AL355149.1-1 and $\operatorname{lnc}$-ZNF674-1 [44]. Interestingly, lncRNAs can be located in whole saliva. HOTAIR is differentially expressed in saliva of metastatic oral squamous cell carcinoma patients compared to primary tumors. These findings suggest that detection of lncRNAs in saliva may be used as a noninvasive and rapid diagnostic tool for diagnosis of oral carcinomas [4].

(ix) Bladder. Oncogenic lncRNAs, including UCA1, H19, $M A L A T 1$, and linc-UBC1 (upregulated in bladder cancer $1)$, are overexpressed in bladder carcinomas and activate PI3K-AKT and Wnt/ $\beta$-catenin pathways. A pilot study took advantage of this to evaluate potential application of UCA1 in urinary sediments from patients with bladder carcinomas. It turned out to be especially valuable for superficial G2/G3 patients at a high risk for muscular invasion, indicating that UCA1 may be a new promising urinary biomarker for the diagnosis of bladder cancer. H19 expression levels are remarkably increased in bladder carcinomas compared to normal tissue and could also serve as another biomarker. More recently, a new lncRNA linc-UBC1 was found to be overexpressed in $60 \%$ of invasive bladder carcinomas and correlated with lymph node metastasis and poor survival. MALAT1 is upregulated in bladder cancer and its expression level is correlated with tumor grade and metastatic stage $[34,35]$.

(x) Prostate. Prostate cancer specific lncRNADD3/PCA3 was identified fifteen years ago [86]. PCA3 is a prostate-specific lncRNA markedly overexpressed in prostate carcinomas. It can be detected in prostate cancer tissue, urine, and/or urine sediments. In recent studies, $P C A 3$ specificity was found even higher than prostate biomarker PSA and PCA3 score can accurately predict tumor volume and pathological features, which may guide treatment $[36,37]$. Therefore, PCA3 can be used as a noninvasive urine-based test for large-scale screening protocols and for predicting prostate carcinomas aggressiveness. This prominent example of rapid translation of lncRNA research into clinical practice offers a prototype for developing different lncRNAs as biomarkers. Other lncRNAs, such as PCGEM1 and PCAT1 (prostate cancer associated ncRNA transcript 1), are also prostate-specific, posing as attractive biomarkers [38, 39].

(xi) Melanoma. BANCR (lncRNA BRAF-activated noncoding RNA) plays a potentially functional role in melanoma cells proliferation and migration by activating ERK1/2 and JNK MAPK pathways. BANCR is upregulated in human malignant melanoma and patients with high levels of expression have a lower survival rate [41].

(xii) Hemopathies. Several recent reports have revealed deregulation of lncRNAs in leukemia, including $A N R I L, \operatorname{lncRNA-}$ P21, MEG3, Dleu2, HOTAIRM1, EGO, and $\operatorname{lncRNA-a7.~More-~}$ over, MEG3, UCA1, and H19 are upregulated in acute myeloid leukemia $[87,88]$.

(xiii) Ovary. HOTAIR plays a pivotal role in epithelial ovarian cancer (EOC) metastasis and could represent a novel prognostic marker and potential therapeutic target in patients with EOC. In recent study, HOTAIR expression was elevated in EOC tissues, and its level of expression is highly positively 
correlated with FIGO stage, histological grade, lymph node metastasis, reduced overall survival, and disease-free survival [33].

Because of their central role in genes expression regulation, lncRNAs could also represent potential therapeutic targets. Better characterization of lncRNAs (structure, functions, polymorphisms, and intracellular topography) could help in faster development of new anticancer strategies modulating expression levels and functions of deregulated lncRNAs. Targeting lncRNAs offers novel exciting opportunity to treat cancer. Currently, nucleic acid-based methods prevail in targeting RNA, by regulating levels of expression and modifying their structures or mature sequences. Among them, RNA interference (RNAi) based techniques are arguably the most popular methods to inhibit lncRNAs in cancer cells. Both siRNAs and shRNAs exhibit great RNA selectivity and knockdown efficiency. Meanwhile, other established methods in inhibiting cancer-associated RNA, including antisense oligonucleotide (ASO), ribozyme, and aptamer, are also effective to modulate lncRNAs, and they show unique features that can have advantages over siRNAs [89].

In principle, targeting of lncRNAs can be achieved using the several approaches, including siRNA-mediated silencing, functional block using small molecules, or oligonucleotide inhibitors to prevent interactions of $\operatorname{lncRNAs}$ with proteins and structure disruption via small molecules or oligonucleotide inhibitors to change or mimic their secondary structure to compete for their binding partners [90].

(1) Small interfering RNAs (siRNA) are short stretched (19-30 nt) double-stranded RNAs that target RNA molecules via complementary to unpaired lncRNA sequences. The RNA duplex of siRNAs must be unwound into single strands before assembling into the active RNA-induced silencing complex (RISC). SiRNAs are fully complementary to their RNA target that is then cleaved at a single phosphodiester bond located near the centre of the sequence complement to the siRNA sequence [90]. SiRNAs exhibit high knockdown efficiency to many oncogenic lncR-NAs in cancer cells and induce anticancer effects both in vitro and in vivo. For instance, depletion of HOTAIR by siRNAs decreases matrix invasiveness of breast cancer cells and inhibits tumor growth of pancreatic cancer xenograft. HULC and MALAT1 siRNAinduced knockdown inhibits HCC cell proliferation and cell cycle progression [91]. Furthermore, siRNA-mediated knockdown of H19 induces apoptosis and inactivates p53 [92]. Phase I and II clinical therapeutic trials have been evaluated using siRNAs to inhibit critical cancer-associated genes, including siRNA-EphA2-DOPC (targeting EphA2), TKM-080301 (targeting PLK1), and CALAA-01 (targeting RRM2). Therapy combining siRNA drug siG12D LODER and Atu027 with conventional chemotherapy is studied in phase II for its therapeutic effect in advanced pancreatic cancer patients. However, the main obstacle of siRNAs therapeutics remaining is their delivery. SiRNAs using ribonucleic acid as building block are susceptible to be degraded by nuclease and have poor pharmacokinetics.

(2) Antisense oligonucleotides (ASO) are short, singlestranded DNAs or RNAs (between 8 and $50 \mathrm{nt}$ ) designed with sequence specific to target lncRNA [90]. ASOs directly hybridize to lncRNA transcripts via base pairing and endogenous RNase $\mathrm{H} 1$, which results in cleavage of lncRNA molecules, then recognizes hybrids formed. ASOs modulate lncRNAs function through degradation of lncRNA transcripts. Inhibition of MALAT1 by ASO attenuates various malignant phenotypes in cancer cells via cycle arrest in cervical cancer cells [93]. Injection of ASO into subcutaneous tumors of nude mice effectively inhibits MALAT1 in vivo and blocks metastasis of lung cancer cells [91].

(3) Ribozymes are naturally produced RNA molecules that present intracellular catalytic functions. One of their functions is degradation of RNA molecules. Among all types of ribozyme, hammerhead ribozyme (HamRz) has caught major interest as it shows good target inhibitory effect while having the smallest RNA endoribonucleolytic motif as well as function independent to presence of metal ions [94]. HamRz is single-stranded RNA in neutral condition and undergoes folding in cells to expose the binding arms. Binding of $\mathrm{HamRz}$ to target sequence depends on complementary match with homologous target site. Both arms of HamRz have to bind with target sites correctly in order to form functional catalytic motif. After binding, HamRz catalyzes cleavage of the flanked RNA region downstream to a NUH site via destabilizing phosphodiester backbone of target RNA [90].

(4) Aptamers are short DNA or RNA oligonucleotides or peptides that have a stable 3-dimensional structure in vivo. They have broad molecular targets including protein, RNA, and small molecules that rely on fitting 3-dimensional shape of their ligands [95]. They specifically bind to their target lncRNAs that rely on fitting 3-dimensional shape of the lncRNA structures. Aptamers antagonize their lncRNA targets by blocking the interactions between lncRNAs and critical factors [90]. Some reports show promising effects of aptamers to either degrade RNA or inhibit RNA functions, implying the potential of aptamers as therapeutic agents to target lncRNAs. Aptamers are used to modulate viral gene expression by interacting with viral RNAs. A hairpin aptamer is identified to form stable and specific complex with the transactivation response element (TAR) RNA element of HIV-1 mRNA and decrease the TAR-dependent viral protein expression [96]. Another aptamer, selected against TAR element, formed stable loop-loop complexes with the element to regulate the TAR-mediated process [97]. Another study showed that aptamer could target the apical loop domain of pri-microRNA molecules and modulate the biogenesis of mature microRNA [98].

(5) MicroRNAs (miRNAs) can interact with lncRNAs. $H 19 / \mathrm{miR}-675$ signaling is critical in glioma progression. By analyzing glioma gene expression data sets, H19 is found to be increased in high grade gliomas. H19 depletion via siRNA inhibits invasion in glioma cells. Further, H19 is positively correlated with its derivate miR-675 expression and reduction of $H 19$ inhibits miR-675 expression. MiR675 modulates cadherin 13 expression by directly targeting its binding site within $3^{\prime}$ UTR. These results demonstrate that $H 19$ regulates glioma development by deriving miR675 and provide important clues for understanding key roles of lncRNA-microRNA functional network in glioma. 
MicroRNA-based anticancer therapy has great potential as reports show there is apparent lack of adverse event in normal tissues when administrated with microRNA-based agent. Various microRNA delivery strategies, such as cationic lipid and nanoparticle encapsulation, are developed to improve microRNA shuttling into target cancer cells $[40,99,100]$. The potential of microRNA regulation on lncRNAs can be further realized by better understanding of intragenic lncRNA regulating elements.

(6) Small molecules are synthesized to specifically bind to RNA binding pockets of lncRNAs. They compete with protein factors or intracellular small ligands for binding lncRNAs. Binding of small molecules may also induce conformational change within lncRNA molecules and disrupt formation of important lncRNA structures [90].

\section{Conclusion and Perspectives}

Deregulated oncogenic and oncosuppressive lncRNAs are observed at all stages of malignant tumors development of various origins. Mechanisms implicating lncRNAs in carcinogenesis are dominated by deregulation of signaling pathways and altered epigenetic, transcriptional, and posttranscriptional expression of numerous genes. Their use as biomarkers and potential therapeutic targets could appear promising.

Major challenges of the next 10 years remaining are identification, mapping of all lncRNAs belonging to the human genome, and their functional characterization. Although their mechanisms of action are better known, it is still possible that lncRNAs are rather downstream products of particular chromatin structures or deregulated transcriptional processes. Their functionality is currently still debated because of their low level of expression and conservation between species. Unlike proteins, which often have welldefined functional areas, it is currently impossible to predict lncRNAs function from their single sequence. However, many traits exhibited by $\operatorname{lncRNAs}$ favor functionality, including $3^{\prime}$ polyadenylation, $5^{\prime}$ cap, multiple exons, transcriptional activation similar to that of mRNA, K4K36 domain, and alternative splicing. These challenges will only be achieved through combined efforts of functional genomics, epigenomics, and bioinformatics.

\section{Conflict of Interests}

No potential conflict of interests was disclosed.

\section{Acknowledgment}

This work was supported by Grant INCa-DGOS-4654.

\section{References}

[1] S. Djebali, C. A. Davis, A. Merkel et al., "Landscape of transcription in human cells," Nature, vol. 489, no. 7414, pp. 101-108, 2012.

[2] M. K. Iyer, Y. S. Niknafs, R. Malik et al., "The landscape of long noncoding RNAs in the human transcriptome," Nature Genetics, vol. 47, no. 3, pp. 199-208, 2015.
[3] R. J. Taft, K. C. Pang, T. R. Mercer, M. Dinger, and J. S. Mattick, "Non-coding RNAs: regulators of disease," Journal of Pathology, vol. 220, no. 2, pp. 126-139, 2010.

[4] E. A. Gibb, K. S. S. Enfield, G. L. Stewart et al., "Long noncoding RNAs are expressed in oral mucosa and altered in oral premalignant lesions," Oral Oncology, vol. 47, no. 11, pp. 10551061, 2011.

[5] J. M. Perkel, "Visiting 'Noncodarnia,' BioTechniques, vol. 54, no. 6, pp. 301-304, 2013.

[6] K. C. Pang, M. C. Frith, and J. S. Mattick, "Rapid evolution of noncoding RNAs: lack of conservation does not mean lack of function," Trends in Genetics, vol. 22, no. 1, pp. 1-5, 2006.

[7] O. Wapinski and H. Y. Chang, "Long noncoding RNAs and human disease," Trends in Cell Biology, vol. 21, no. 6, pp. 354$361,2011$.

[8] S. Ye, L. Yang, X. Zhao, W. Song, W. Wang, and S. Zheng, "Bioinformatics method to predict two regulation mechanism: TF-miRNA-mRNA and lncRNA-miRNA-mRNA in pancreatic cancer," Cell Biochemistry and Biophysics, vol. 70, no. 3, pp. 1849-1858, 2014.

[9] Q. Jiang, J. Wang, Y. Wang, R. Ma, X. Wu, and Y. Li, "TF2LncRNA: identifying common transcription factors for a list of lncRNA genes from ChIP-seq data," BioMed Research International, vol. 2014, Article ID 317642, 5 pages, 2014.

[10] C. Park, N. Yu, I. Choi, W. Kim, and S. Lee, "IncRNAtor: a comprehensive resource for functional investigation of long non-coding RNAs," Bioinformatics, vol. 30, no. 17, pp. 24802485, 2014.

[11] Z. X. Chen, D. Sturgill, J. Qu et al., "Comparative validation of the D. melanogaster modENCODE transcriptome annotation," Genome Research, vol. 24, no. 7, pp. 1209-1223, 2014.

[12] Z. Chen, J. Li, L. Tian et al., "MiRNA expression profile reveals a prognostic signature for esophageal squamous cell carcinoma," Cancer Letters, vol. 350, no. 1-2, pp. 34-42, 2014.

[13] M. Hajjari, M. Behmanesh, M. Sadeghizadeh, and M. Zeinoddini, "Up-regulation of HOTAIR long non-coding RNA in human gastric adenocarcinoma tissues," Medical Oncology, vol. 30 , no. 3, article $670,2013$.

[14] F. Yang, X. Xue, J. Bi et al., "Long noncoding RNA CCAT1, which could be activated by c-Myc, promotes the progression of gastric carcinoma," Journal of Cancer Research and Clinical Oncology, vol. 139, no. 3, pp. 437-445, 2013.

[15] W. Sun, Y. Wu, X. Yu et al., "Decreased expression of long noncoding RNA AC096655.1-002 in gastric cancer and its clinical significance," Tumor Biology, vol. 34, no. 5, pp. 26972701, 2013.

[16] B. Xiao and J. Guo, "Long noncoding RNA AC096655.1-002 has been officially named as gastric cancer-associated transcript 1 , GACAT1," Tumor Biology, vol. 34, no. 5, p. 3271, 2013.

[17] D. Mei, H. Song, K. Wang et al., "Up-regulation of SUMO1 pseudogene 3 (SUMO1P3) in gastric cancer and its clinical association," Medical Oncology, vol. 30, no. 4, article 709, 2013.

[18] J. Sana, P. Faltejskova, M. Svoboda, and O. Slaby, "Novel classes of non-coding RNAs and cancer," Journal of Translational Medicine, vol. 10, no. 1, article 103, 2012.

[19] R. Kogo, T. Shimamura, K. Mimori et al., "Long noncoding RNA HOTAIR regulates polycomb-dependent chromatin modification and is associated with poor prognosis in colorectal cancers," Cancer Research, vol. 71, no. 20, pp. 6320-6326, 2011.

[20] K. Panzitt, M. M. O. Tschernatsch, C. Guelly et al., "Characterization of HULC, a novel gene with striking up-regulation in 
hepatocellular carcinoma, as noncoding RNA," Gastroenterology, vol. 132, no. 1, pp. 330-342, 2007.

[21] H. Xie, H. Ma, and D. Zhou, "Plasma HULC as a promising novel biomarker for the detection of hepatocellular carcinoma," BioMed Research International, vol. 2013, Article ID 136106, 5 pages, 2013.

[22] J.-F. Huang, Y.-J. Guo, C.-X. Zhao et al., "Hepatitis B virus X protein (HBx)-related long noncoding RNA (lncRNA) downregulated expression by $\mathrm{HBx}$ (Dreh) inhibits hepatocellular carcinoma metastasis by targeting the intermediate filament protein vimentin," Hepatology, vol. 57, no. 5, pp. 1882-1892, 2013.

[23] T. Gutschner, M. Hämmerle, M. Eissmann et al., “The noncoding RNA MALAT1 is a critical regulator of the metastasis phenotype of lung cancer cells," Cancer Research, vol. 73, no. 3, pp. 1180-1189, 2013.

[24] E. B. Zhang, D. D. Yin, M. Sun et al., "P53-regulated long noncoding RNA TUG1 affects cell proliferation in human nonsmall cell lung cancer, partly through epigenetically regulating HOXB7 expression," Cell Death and Disease, vol. 5, no. 5, Article ID e1243, 2014.

[25] T. Nakagawa, H. Endo, M. Yokoyama et al., "Large noncoding RNA HOTAIR enhances aggressive biological behavior and is associated with short disease-free survival in human nonsmall cell lung cancer," Biochemical and Biophysical Research Communications, vol. 436, no. 2, pp. 319-324, 2013.

[26] J. Yang, J. Lin, T. Liu et al., "Analysis of lncRNA expression profiles in non-small cell lung cancers (NSCLC) and their clinical subtypes," Lung Cancer, vol. 85, no. 2, pp. 110-115, 2014.

[27] M. Sun, X.-H. Liu, K.-M. Wang et al., "Downregulation of BRAF activated non-coding RNA is associated with poor prognosis for non-small cell lung cancer and promotes metastasis by affecting epithelial-mesenchymal transition," Molecular Cancer, vol. 13, no. 1, article 68, 2014.

[28] A. Renganathan, J. Kresoja-Rakic, N. Echeverry et al., "GAS5 long non-coding RNA in malignant pleural mesothelioma," Molecular Cancer, vol. 13, no. 1, article 119, 2014.

[29] K. M. Chisholm, Y. Wan, R. Li, K. D. Montgomery, H. Y. Chang, and R. B. West, "Detection of long non-coding RNA in archival tissue: correlation with polycomb protein expression in primary and metastatic breast carcinoma," PLoS ONE, vol. 7, no. 10, Article ID e47998, 2012.

[30] A. Bhan, I. Hussain, K. I. Ansari, S. Kasiri, A. Bashyal, and S. S. Mandal, "Antisense transcript long noncoding RNA (lncRNA) HOTAIR is transcriptionally induced by estradiol," Journal of Molecular Biology, vol. 425, no. 19, pp. 3707-3722, 2013.

[31] L. Lu, G. Zhu, C. Zhang et al., "Association of large noncoding RNA HOTAIR expression and its downstream intergenic CpG island methylation with survival in breast cancer," Breast Cancer Research and Treatment, vol. 136, no. 3, pp. 875-883, 2012.

[32] J. Huang, N. Zhou, K. Watabe et al., "Long non-coding RNA UCA1 promotes breast tumor growth by suppression of p27 (Kip1)," Cell Death and Disease, vol. 5, no. 1, p. e1008, 2014.

[33] J.-J. Qiu, Y.-Y. Lin, L.-C. Ye et al., “Overexpression of long non-coding RNA HOTAIR predicts poor patient prognosis and promotes tumor metastasis in epithelial ovarian cancer," Gynecologic Oncology, vol. 134, no. 1, pp. 121-128, 2014.

[34] Q. Zhang, M. Su, G. Lu, and J. Wang, "The complexity of bladder cancer: long noncoding RNAs are on the stage," Molecular Cancer, vol. 12, no. 1, article 101, 2013.

[35] L. Wang, D. Fu, Y. Qiu et al., "Genome-wide screening and identification of long noncoding RNAs and their interaction with protein coding RNAs in bladder urothelial cell carcinoma," Cancer Letters, vol. 349, no. 1, pp. 77-86, 2014.

[36] G. Ploussard, X. Durand, E. Xylinas et al., "Prostate cancer antigen 3 score accurately predicts tumour volume and might help in selecting prostate cancer patients for active surveillance," European Urology, vol. 59, no. 3, pp. 422-429, 2011.

[37] M. J. Roobol, F. H. Schröder, G. L. J. H. Van Leenders et al., "Performance of prostate cancer antigen 3 (PCA3) and prostate-specific antigen in prescreened men: Reproducibility and detection characteristics for prostate cancer patients with high PCA3 scores ( $\geq 100)$," European Urology, vol. 58, no. 6, pp. 893-899, 2010.

[38] A. L. Walsh, A. V. Tuzova, E. M. Bolton, T. H. Lynch, and A. S. Perry, "Long noncoding RNAs and prostate carcinogenesis: the missing 'linc'?" Trends in Molecular Medicine, vol. 20, no. 8, pp. 428-436, 2014.

[39] G. O. Ifere and G. A. Ananaba, "Prostate Cancer Gene Expression Marker 1 (PCGEM1): a patented prostate- specific noncoding gene and regulator of prostate cancer progression," Recent Patents on DNA and Gene Sequences, vol. 3, no. 3, pp. 151-163, 2009.

[40] Y. Shi, Y. Wang, W. Luan et al., "Long non-coding RNA H19 promotes glioma cell invasion by deriving miR-675," PLoS ONE, vol. 9, no. 1, Article ID e86295, 2014.

[41] R. Li, L. Zhang, L. Jia et al., "Long non-coding RNA BANCR promotes proliferation in malignant melanoma by regulating MAPK pathway activation," PLoS ONE, vol. 9, no. 6, Article ID e100893, 2014.

[42] R. A. Gupta, N. Shah, K. C. Wang et al., "Long non-coding RNA HOTAIR reprograms chromatin state to promote cancer metastasis," Nature, vol. 464, no. 7291, pp. 1071-1076, 2010.

[43] Y. Nie, X. Liu, S. Qu, E. Song, H. Zou, and C. Gong, "Long noncoding RNA HOTAIR is an independent prognostic marker for nasopharyngeal carcinoma progression and survival," Cancer Science, vol. 104, no. 4, pp. 458-464, 2013.

[44] W. Gao, J. Y.-W. Chan, and T.-S. Wong, "Differential expression of long noncoding RNA in primary and recurrent nasopharyngeal carcinoma," BioMed Research International, vol. 2014, Article ID 404567, 9 pages, 2014.

[45] A. C. Marques, J. Hughes, B. Graham, M. S. Kowalczyk, D. R. Higgs, and C. P. Ponting, "Chromatin signatures at transcriptional start sites separate two equally populated yet distinct classes of intergenic long noncoding RNAs," Genome Biology, vol. 14, no. 11, article R131, 2013.

[46] R. Louro, A. S. Smirnova, and S. Verjovski-Almeida, "Long intronic noncoding RNA transcription: expression noise or expression choice?” Genomics, vol. 93, no. 4, pp. 291-298, 2009.

[47] T. Kunej, J. Obsteter, Z. Pogacar, S. Horvat, and G. A. Calin, “The decalog of long non-coding RNA involvement in cancer diagnosis and monitoring," Critical Reviews in Clinical Laboratory Sciences, vol. 51, no. 6, pp. 344-357, 2014.

[48] A. M. Khalil, M. Guttman, M. Huarte et al., "Many human large intergenic noncoding RNAs associate with chromatinmodifying complexes and affect gene expression," Proceedings of the National Academy of Sciences of the United States of America, vol. 106, no. 28, pp. 11667-11672, 2009.

[49] W. Xie and B. Ren, "Enhancing pluripotency and lineage specification," Science, vol. 341, no. 6143, pp. 245-247, 2013.

[50] S. Nakagawa, J. Y. Ip, G. Shioi et al., "Malatl is not an essential component of nuclear speckles in mice," RNA, vol. 18, no. 8, pp. 1487-1499, 2012. 
[51] I. Ilik, J. Quinn, P. Georgiev et al., "Tandem stem-loops in roX RNAs act together to mediate X Chromosome dosage compensation in Drosophila," Molecular Cell, vol. 51, no. 2, pp. 156-173, 2013.

[52] Y. A. Sytnikova, R. Rahman, G. Chirn, J. P. Clark, and N. C. Lau, "Transposable element dynamics and PIWI regulation impacts lncRNA and gene expression diversity in Drosophila ovarian cell cultures," Genome Research, vol. 24, no. 12, pp. 1977-1990, 2014.

[53] J. W. Nam and D. P. Bartel, "Long noncoding RNAs in C. elegans," Genome Research, vol. 22, no. 12, pp. 2529-2540, 2012.

[54] Y. Zhang, J. Xia, Q. Li et al., "NRF2/long noncoding RNA ROR signaling regulates mammary stem cell expansion and protects against estrogen genotoxicity," The Journal of Biological Chemistry, vol. 289, no. 45, pp. 31310-31318, 2014.

[55] S. He, H. Zhang, H. Liu, and H. Zhu, "LongTarget: a tool to predict lncRNA DNA-binding motifs and binding sites via Hoogsteen base-pairing analysis," Bioinformatics, vol. 31, no. 2, pp. 178-186, 2015.

[56] J. C. Schwartz, S. T. Younger, N.-B. Nguyen et al., "Antisense transcripts are targets for activating small RNAs," Nature Structural and Molecular Biology, vol. 15, no. 8, pp. 842-848, 2008.

[57] R. J. Taft, C. D. Kaplan, C. Simons, and J. S. Mattick, "Evolution, biogenesis and function of promoter-associated RNAs," Cell Cycle, vol. 8, no. 15, pp. 2332-2338, 2009.

[58] J. Han, D. Kim, and K. V. Morris, "Promoter-associated RNA is required for RNA-directed transcriptional gene silencing in human cells," Proceedings of the National Academy of Sciences of the United States of America, vol. 104, no. 30, pp. 12422-12427, 2007.

[59] S. Memczak, M. Jens, A. Elefsinioti et al., "Circular RNAs are a large class of animal RNAs with regulatory potency," Nature, vol. 495, no. 7441, pp. 333-338, 2013.

[60] K. C. Wang and H. Y. Chang, "Molecular mechanisms of long noncoding RNAs," Molecular Cell, vol. 43, no. 6, pp. 904-914, 2011.

[61] E. Leygue, H. Dotzlaw, P. H. Watson, and L. C. Murphy, "Expression of the steroid receptor RNA activator in human breast tumors," Cancer Research, vol. 59, no. 17, pp. 4190-4193, 1999.

[62] S. Chooniedass-Kothari, E. Emberley, M. K. Hamedani et al., "The steroid receptor RNA activator is the first functional RNA encoding a protein," FEBS Letters, vol. 566, no. 1-3, pp. 43-47, 2004.

[63] D. Ulveling, C. Francastel, and F. Hubé, "When one is better than two: RNA with dual functions," Biochimie, vol. 93, no. 4, pp. 633-644, 2011.

[64] J. L. Rinn, M. Kertesz, J. K. Wang et al., "Functional demarcation of active and silent chromatin domains in human HOX Loci by noncoding RNAs," Cell, vol. 129, no. 7, pp. 1311-1323, 2007.

[65] M.-C. Tsai, O. Manor, Y. Wan et al., "Long noncoding RNA as modular scaffold of histone modification complexes," Science, vol. 329, no. 5992, pp. 689-693, 2010.

[66] E. Pasmant, I. Laurendeau, D. Héron, M. Vidaud, D. Vidaud, and I. Bièche, "Characterization of a germ-line deletion, including the entire INK4/ARF locus, in a melanoma-neural system tumor family: identification of ANRIL, an antisense noncoding RNA whose expression coclusters with ARF," Cancer Research, vol. 67, no. 8, pp. 3963-3969, 2007.

[67] E. Pasmant, A. Sabbagh, J. Masliah-Planchon et al., "Role of noncoding RNA ANRIL in genesis of plexiform neurofibromas in neurofibromatosis type 1," Journal of the National Cancer Institute, vol. 103, no. 22, pp. 1713-1722, 2011.
[68] E. Pasmant, A. Sabbagh, M. Vidaud, and I. Bièche, "ANRIL, a long, noncoding RNA, is an unexpected major hotspot in GWAS," FASEB Journal, vol. 25, no. 2, pp. 444-448, 2011.

[69] L. Shen, L. Chen, Y. Wang, X. Jiang, H. Xia, and Z. Zhuang, "Long noncoding RNA MALAT1 promotes brain metastasis by inducing epithelial-mesenchymal transition in lung cancer," Journal of Neuro-Oncology, vol. 121, no. 1, pp. 101-108, 2015.

[70] J. Wang, X. Liu, H. Wu et al., "CREB up-regulates long non-coding RNA, HULC expression through interaction with microRNA-372 in liver cancer," Nucleic Acids Research, vol. 38, no. 16, pp. 5366-5383, 2010.

[71] J. H. He, J. Z. Zhang, Z. P. Han, L. Wang, Y. Lv, and Y. G. $\mathrm{Li}$, "Reciprocal regulation of PCGEM1 and miR-145 promote proliferation of LNCaP prostate cancer cells," Journal of Experimental \& Clinical Cancer Research, vol. 33, no. 1, article 72, 2014.

[72] K.-H. Lu, W. Li, X.-H. Liu et al., "Long non-coding RNA MEG3 inhibits NSCLC cells proliferation and induces apoptosis by affecting p53 expression," BMC Cancer, vol. 13, article 461, 2013.

[73] M. R. Pickard and G. T. Williams, "Regulation of apoptosis by long non-coding RNA GAS5 in breast cancer cells: implications for chemotherapy," Breast Cancer Research and Treatment, vol. 145, no. 2, pp. 359-370, 2014.

[74] M. R. Pickard, M. Mourtada-Maarabouni, and G. T. Williams, "Long non-coding RNA GAS5 regulates apoptosis in prostate cancer cell lines," Biochimica et Biophysica Acta, vol. 1832, no. 10, pp. 1613-1623, 2013.

[75] R. Kurokawa, "Promoter-associated long noncoding RNAs repress transcription through a RNA binding protein TLS," Advances in Experimental Medicine and Biology, vol. 722, pp. 196-208, 2011.

[76] X. Song, X. Wang, S. Arai, and R. Kurokawa, "Promoterassociated noncoding RNA from the CCND1 promoter," Methods in Molecular Biology, vol. 809, pp. 609-622, 2012.

[77] G. Wu, J. Cai, Y. Han et al., "LincRNA-p21 regulates neointima formation, vascular smooth muscle cell proliferation, apoptosis, and atherosclerosis by enhancing p53 activity," Circulation, vol. 130, no. 17, pp. 1452-1465, 2014.

[78] V. Pfeiffer and J. Lingner, “TERRA promotes telomere shortening through exonuclease 1-mediated resection of chromosome ends," PLoS Genetics, vol. 8, no. 6, Article ID e1002747, 2012.

[79] M. Nordin, D. Bergman, M. Halje, W. Engström, and A. Ward, "Epigenetic regulation of the Igf2/H19 gene cluster," Cell Proliferation, vol. 47, no. 3, pp. 189-199, 2014.

[80] S. Marquardt, R. Escalante-Chong, N. Pho et al., "A chromatinbased mechanism for limiting divergent noncoding transcription," Cell, vol. 157, no. 7, pp. 1712-1723, 2014.

[81] R. Backofen and T. Vogel, "Biological and bioinformatical approaches to study crosstalk of long-non-coding RNAs and chromatin-modifying proteins," Cell and Tissue Research, vol. 356, no. 3, pp. 507-526, 2014.

[82] M.-C. Lai, Z. Yang, L. Zhou et al., "Long non-coding RNA MALAT-1 overexpression predicts tumor recurrence of hepatocellular carcinoma after liver transplantation," Medical Oncology, vol. 29, no. 3, pp. 1810-1816, 2012.

[83] Z. Yang, L. Zhou, L.-M. Wu et al., "Overexpression of long noncoding RNA HOTAIR predicts tumor recurrence in hepatocellular carcinoma patients following liver transplantation," Annals of Surgical Oncology, vol. 18, no. 5, pp. 1243-1250, 2011.

[84] L. Quagliata, M. S. Matter, S. Piscuoglio et al., "Long noncoding RNA HOTTIP/HOXA13 expression is associated with disease progression and predicts outcome in hepatocellular carcinoma patients," Hepatology, vol. 59, no. 3, pp. 911-923, 2014. 
[85] S.-X. Yuan, F. Yang, Y. Yang et al., "Long noncoding RNA associated with microvascular invasion in hepatocellular carcinoma promotes angiogenesis and serves as a predictor for hepatocellular carcinoma patients' poor recurrence-free survival after hepatectomy," Hepatology, vol. 56, no. 6, pp. 2231-2241, 2012.

[86] M. J. G. Bussemakers, A. Van Bokhoven, G. W. Verhaegh et al., "DD3: a new prostate-specific gene, highly overexpressed in prostate cancer," Cancer Research, vol. 59, no. 23, pp. 5975-5979, 1999.

[87] M. Hajjari, A. Khoshnevisan, and Y. K. Shin, "Long noncoding RNAs in hematologic malignancies: road to translational research," Frontiers in Genetics, vol. 4, article 250, 2013.

[88] E. F. Heuston, K. T. Lemon, and R. J. Arceci, “The beginning of the road for non-coding RNAs in normal hematopoiesis and hematologic malignancies," Frontiers in Genetics, vol. 2, article 94, 2011.

[89] C. H. Li and Y. Chen, "Targeting long non-coding RNAs in cancers: progress and prospects," International Journal of Biochemistry and Cell Biology, vol. 45, no. 8, pp. 1895-1910, 2013.

[90] L. Ma, M.-S. Chua, O. Andrisani, and S. So, "Epigenetics in hepatocellular carcinoma: an update and future therapy perspectives," World Journal of Gastroenterology, vol. 20, no. 2, pp. 333-345, 2014.

[91] T. Gutschner, M. Hämmerle, and S. Diederichs, "MALAT1—a paradigm for long noncoding RNA function in cancer," Journal of Molecular Medicine, vol. 91, no. 7, pp. 791-801, 2013.

[92] F. Yang, J. Bi, X. Xue et al., "Up-regulated long non-coding RNA H19 contributes to proliferation of gastric cancer cells," FEBS Journal, vol. 279, no. 17, pp. 3159-3165, 2012.

[93] V. Tripathi, Z. Shen, A. Chakraborty et al., "Long noncoding RNA MALAT1 controls cell cycle progression by regulating the expression of oncogenic transcription factor B-MYB," PLoS Genetics, vol. 9, no. 3, Article ID e1003368, 2013.

[94] D. E. Ruffner, G. D. Stormo, and O. C. Uhlenbeck, "Sequence requirements of the hammerhead RNA self-cleavage reaction," Biochemistry, vol. 29, no. 47, pp. 10695-10702, 1990.

[95] J. Müller, O. El-Maarri, J. Oldenburg, B. Pötzsch, and G. Mayer, "Monitoring the progression of the in vitro selection of nucleic acid aptamers by denaturing high-performance liquid chromatography," Analytical and Bioanalytical Chemistry, vol. 390, no. 4, pp. 1033-1037, 2008.

[96] G. Kolb, S. Reigadas, D. Castanotto et al., "Endogenous expression of an anti-TAR aptamer reduces HIV-1 replication," RNA Biology, vol. 3, no. 4, pp. 150-156, 2006.

[97] M. Watrin, F. von Pelchrzim, E. Dausse, R. Schroeder, and J.J. Toulmé, "In vitro selection of RNA aptamers derived from a genomic human library against the TAR RNA element of HIV1," Biochemistry, vol. 48, no. 26, pp. 6278-6284, 2009.

[98] C. E. Lünse, G. Michlewski, C. S. Hopp et al., "An aptamer targeting the apical-loop domain modulates pri-miRNA processing," Angewandte Chemie-International Edition, vol. 49, no. 27, pp. 4674-4677, 2010.

[99] Y. Wu, Y. Xiao, X. Ding et al., "A mir-200b/200c/429-binding site polymorphism in the $3^{\prime}$ untranslated region of the AP- $2 \alpha$ gene is associated with cisplatin resistance," PLoS ONE, vol. 6, no. 12, Article ID e29043, 2011.

[100] J. Wu, G. Wu, L. Lv et al., "MicroRNA-34a inhibits migration and invasion of colon cancer cells via targeting to Fra-1," Carcinogenesis, vol. 33, no. 3, pp. 519-528, 2012. 


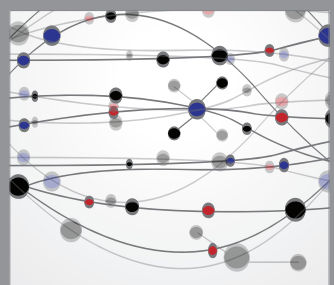

The Scientific World Journal
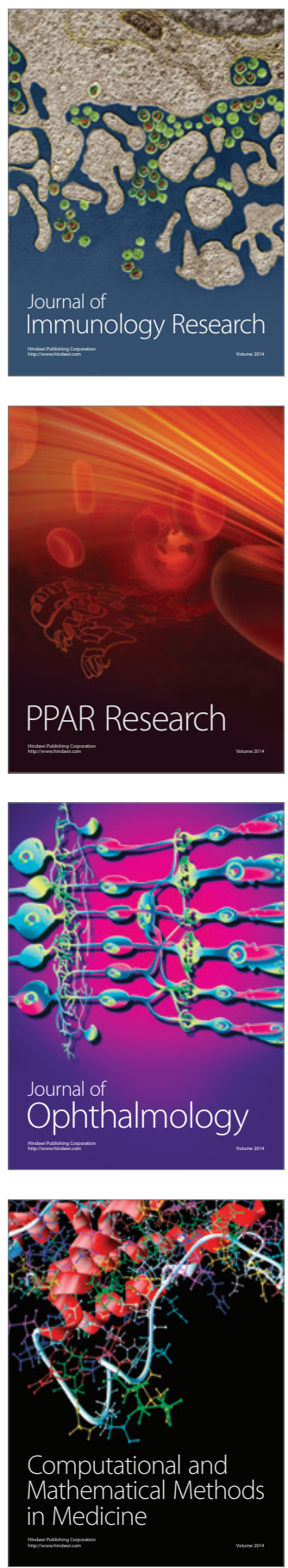

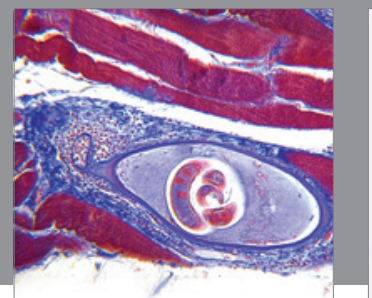

Gastroenterology

Research and Practice
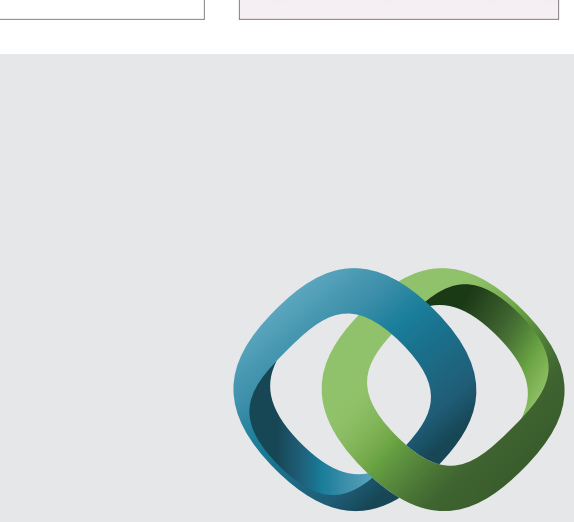

\section{Hindawi}

Submit your manuscripts at

http://www.hindawi.com
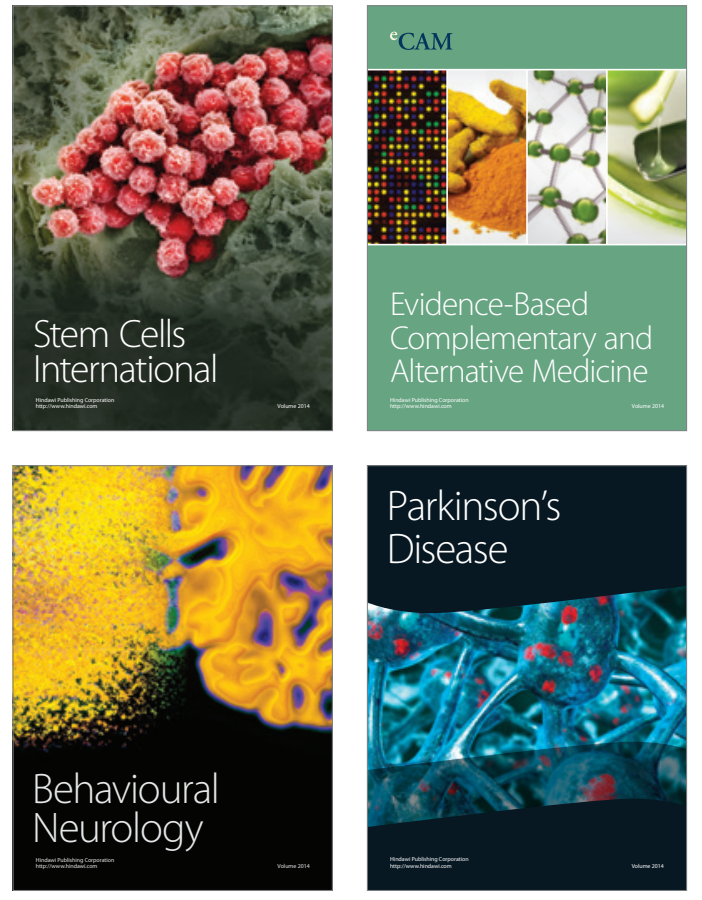
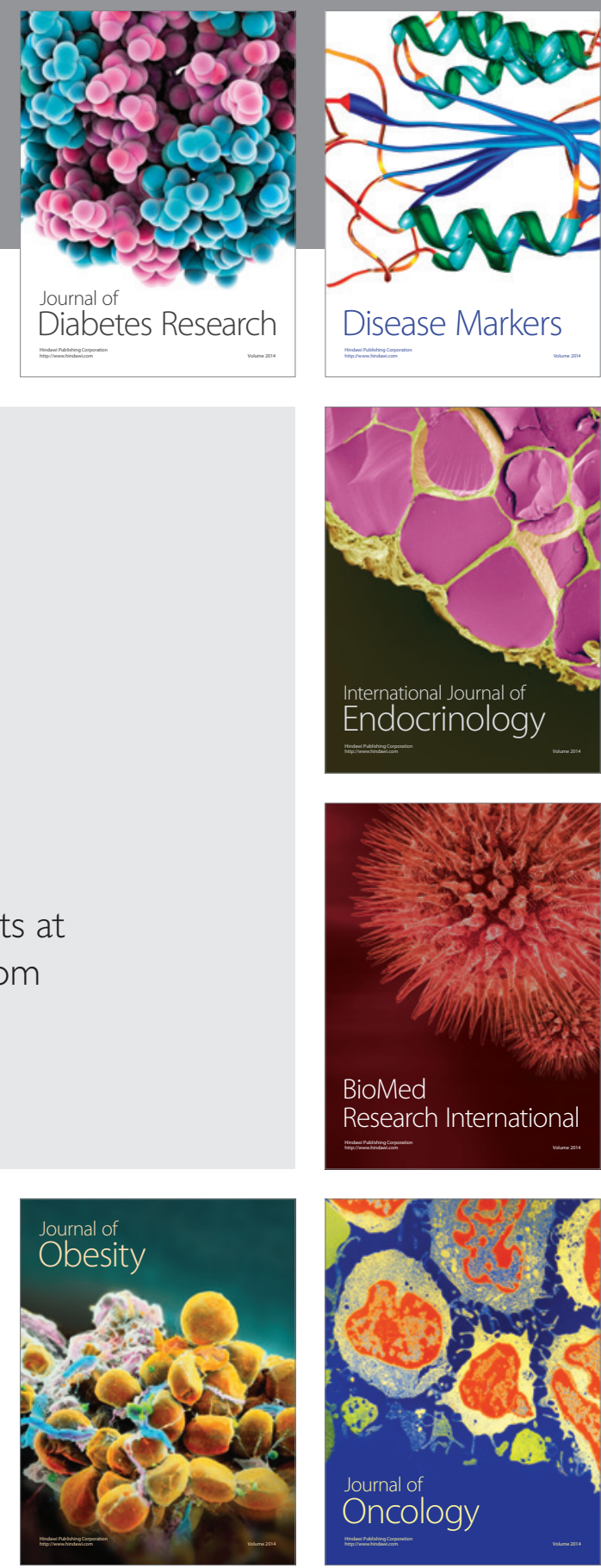

Disease Markers
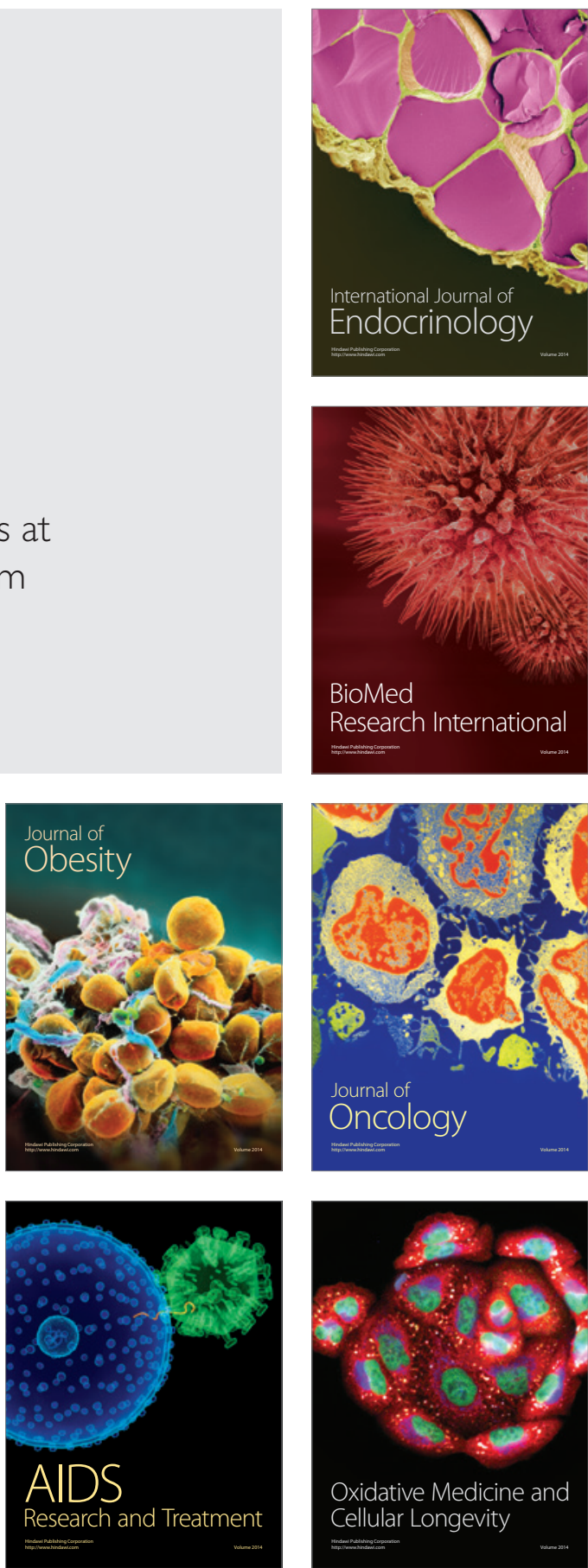